\title{
Enhanced Liver Regeneration in IL-10-Deficient Mice after Partial Hepatectomy via Stimulating Inflammatory Response and Activating Hepatocyte STAT3
}

\author{
Shi Yin, ${ }^{* \dagger \S}$ Hua Wang, ${ }^{\dagger \uparrow}$ Ogyi Park, ${ }^{\dagger}$ Wei Wei, ${ }^{*}$ \\ Jilong Shen, ${ }^{* £ \S}$ and Bin $\mathrm{Gao}^{\dagger}$ \\ From the Institute of Clinical Pharmacology, ${ }^{*}$ the Provincial \\ Laboratory of Microbiology \& Parasitology, Ministry of \\ Education, and the Departments of Microbiology and \\ Parasitology, and Oncology, ${ }^{\top}$ Anhui Medical University, Anbui, \\ China; and the Laboratory of Liver Diseases, ${ }^{\dagger}$ National Institute \\ on Alcohol Abuse and Alcoholism, National Institutes of Health, \\ Bethesda, Maryland
}

Emerging evidence suggests that proinflammatory cytokines, including tumor necrosis factor- $\alpha$ (TNF- $\alpha$ ) and interleukin-6 (IL-6), play a critical role in the initiation and progression of liver regeneration; however, relatively little is known about the role of antiinflammatory cytokine IL-10 in liver regeneration after partial hepatectomy (PHx). Here, we examined the role of IL-10 in liver regeneration using a model of PHx in several strains of genetically modified mice. After PHx, expression of IL-10 mRNA in the liver and spleen was significantly elevated. Such elevation was diminished in TLR4 mutant mice. Compared with wild-type mice, $\mathrm{IL}^{-10^{-/-}}$mice had higher levels of expression of proinflammatory cytokines (IL-6, TNF- $\alpha$, and IFN- $\gamma$ ) and inflammatory markers (CCR2 and $\mathrm{F} 4 / 80$ ) in the liver, as well as higher serum levels of proinflammatory cytokines after PHx. The number of neutrophils and macrophages was also higher in the livers of $\mathrm{IL}^{-10^{-/-}}$mice than in wild-type mice after PHx. Liver regeneration as determined by BrdU incorporation after PHx was higher in $\mathrm{IL}^{-10^{-/-}}$mice than in wild-type mice, which was associated with higher levels of activation of IL- 6 downstream signal STAT3 in the liver. An additional deletion of STAT3 in hepatocytes significantly reduced liver regeneration in IL-10 $^{-/-}$mice after PHx. Collectively, IL-10 plays an important role in negatively regulating liver regeneration via limiting inflammatory response and subsequently tempering hepatic STAT3 activation. (Am J Pathol 2011, 178:1614-1621; DOI: 10.1016/j.ajpath.2011.01.001)
The liver is the only solid organ in mammals with remarkable regenerative capabilities. The differentiated hepatocytes that are normally quiescent can re-enter the cell cycle in response to tissue loss or injury and divide until the original liver mass is restored. ${ }^{1-6}$ Two-thirds partial hepatectomy $(\mathrm{PHx})$ represents the most commonly used model for the study of liver regeneration. Liver regeneration involves a sequence of signaling events with highly synchronized cell cycle profile to restore liver mass and function. After two-thirds PHx in mice, hepatocytes are the first type of liver cells to start proliferating and undergo one to two rounds of cell division, with DNA synthesis starting within 24 hours, and proliferation peaks (S phase) at around 36 to 42 hours after surgery. Restoration of liver mass is nearly complete by 7 to 10 days in rodents and by 3 to 4 months in humans. ${ }^{1-6}$

Liver regeneration involves a multiplicity of pathways and cellular proliferation kinetics. Much is now understood about the role of cytokines, growth factors, hormones, and their downstream signals in driving and terminating the liver regeneration process. ${ }^{1-7}$ Among them, the two important proinflammatory cytokines TNF- $\alpha$ and IL-6, which are critical contributors to innate and adaptive immune responses, are well established as regenerationpromoting cytokines in the priming phase and progress of liver regeneration. ${ }^{1-6}$ It is widely accepted that after $\mathrm{PHx}$, the ability of the remnant liver to detoxify endotoxin (LPS) decreases, leading to elevation of hepatic LPS

Supported in part by the intramural program of National Institute on Alcohol Abuse and Alcoholism of the National Institutes of Health (NIH) (B.G.) and in part by the Natural Science Foundation of China (No. 30973467/H1611 to H.W.).

This work is also a part of Shi Yin's PhD thesis work from the Graduate Partner Program (GPP) between NIH and Anhui Medical University. This work was presented orally by Shi Yin at the 97th American Association of Immunologists Annual Meeting in Baltimore, MD, in May 2010.

Accepted for publication January 4, 2011

Address reprint requests to Bin Gao, M.D., Ph.D., Laboratory of Liver Diseases, National Institute on Alcohol Abuse and Alcoholism, National Institutes of Health, Bethesda, MD 20892; or Jilong Shen, Ph.D., Department of Microbiology and Parasitology, Anhui Medical University, Hefei, Anhui 230032, China. E-mail: bgao@mail.nih.gov or jlshen@ahmu.edu.cn. 
levels. ${ }^{8,9}$ LPS then stimulates Kupffer cells to produce TNF- $\alpha$ and IL-6, which subsequently initiate liver regeneration. ${ }^{1-6,10,11}$ The proinflammatory cytokine TNF- $\alpha$ and its downstream signaling molecule NF- $\kappa \mathrm{B}$ have been shown to play a particularly important role in the earliest step of priming of hepatocytes and stimulation of hepatocyte proliferation via induction of IL-6 during liver regeneration. ${ }^{11}$ The action of IL-6 is mediated via binding the IL-6 receptor complex (gp80/gp130) on hepatocytes, followed by activation of STAT3 and promoting hepatocyte survival and proliferation. ${ }^{12-16}$ The findings from these published reports suggest that the inflammatory response and cytokine production are particularly active during the first 24 hours after $\mathrm{PHx}$, a time when hepatocyte transition from a quiescent state into the cell cycle occurs. However, how these inflammatory responses are controlled during liver regeneration remains largely unknown. In the current study, we have identified IL-10, a potent anti-inflammatory cytokine, ${ }^{17}$ as an important regulator to suppress liver inflammation and regeneration after $\mathrm{PHx}$ via regulating production of proinflammatory cytokines and subsequently suppressing STAT3 activation in the liver.

\section{Materials and Methods}

\section{Animals}

Eight- to 10-week-old male mice were used in this study. IL-10 ${ }^{-/-}$mice and their wild-type control C57BL/6J mice, and TLR4 mutant mice and their wild-type control mice were purchased from the Jackson Laboratory (Bar Harbor, ME). Hepatocyte-specific STAT3 knockout (KO) (STAT3 ${ }^{\mathrm{Hep}-1-}$ ) mice were described previously. ${ }^{18}$ Male IL-10 $0^{-1-}$ were bred with female STAT3 ${ }^{\mathrm{Hep}-1-}$ mice for several steps to generate $\mathrm{IL}-10^{-1-}$ STAT3 ${ }^{\text {Hep- }-1-}$ mice in which the STAT3 gene was deleted in hepatocytes, whereas the IL-10 gene was deleted globally. All knockout strains mentioned above were developmentally normal and have normal life spans. All animal studies were approved by the Institutional Animal Care and Use Committees of the National Institute on Alcohol Abuse and Alcoholism and the National Institutes of Health.

\section{Partial Hepatectomy Model}

Mice were anesthetized by inhalation of isoflurane (2\%), followed by ligation of the median and left lateral lobes of the liver at their stem, and excision under aseptic conditions, as described previously. ${ }^{19}$ For sham operation, mice were anesthetized and then subject to laparotomy, followed by brief manipulation of the intestines, but not the liver, with cotton swabs before wound closure. The animals were sacrificed at the indicated times following surgery. Mortality was less than $5 \%$ and not associated with a particular genotype.

\section{Determination of the Rate of Liver Regeneration}

Liver regeneration rate was determined by the BrdU incorporation assay, and mitotic hepatocytes were counted. Briefly, partially hepatectomized mice were injected intraperitoneally with $\mathrm{BrdU}$ (50 $\mu \mathrm{g} / \mathrm{g}$ body weight) at various time points after partial hepatectomy $(\mathrm{PHx})$. Mice were euthanized 2 hours after BrdU injection, and the livers were harvested and fixed in $10 \%$ neutral buffered formalin for 24 hours. Fixed livers were embedded in paraffin, cut into $5-\mu \mathrm{m}$ tissue sections by a microtome, and then adhered to poly-L-lysine-coated glass slides. The slides were dried overnight at $37^{\circ} \mathrm{C}$. BrdU incorporation was visualized immunohistochemically using a BrdU immunostaining kit (BD Biosciences, San Diego, $\mathrm{CA}$ ) according to the manufacturer's instructions. BrdUlabeled hepatocytes were quantified by counting positively stained hepatocyte nuclei in three to six low-power $(\times 100)$ microscope fields, and the means were calculated. For mitotic hepatocytes, at least 500 hepatocytes were counted for mitotic positivity at least three times in different sections in each group. Mitotic figures are calculated as fractions of the total number of hepatocytes examined (number of mitotic hepatocytes/total number of hepatocytes \%).

\section{Histology}

Formalin-fixed liver samples were processed, and paraffin sections of $5-\mu \mathrm{m}$ thickness were stained with hematoxylin and eosin (H\&E). Inflammatory foci were counted in 10 randomly selected fields ( $\times 100$ magnification).

\section{Cytokine Levels}

Cytokine levels in the serum and liver were measured using cytometry bead array (BD Biosciences) according to the manufacturer's protocol. The analysis was conducted using a conventional flow cytometer (FACScalibur; Becton Dickinson, Mountain View, CA).

\section{Western Blotting}

Liver homogenates were prepared in RIPA buffer (50 $\mathrm{mmol} / \mathrm{L}$ Tris; $1 \%$ NP40; 0.25\% deoxycholic acid sodium salt; $150 \mathrm{mmol} / \mathrm{L} \mathrm{NaCl} ; 1 \mathrm{mmol} / \mathrm{L}$ EGTA) containing $1 \mathrm{mmol} / \mathrm{L}$ $\mathrm{Na}_{3} \mathrm{VO}_{4}$ and a protease inhibitor cocktail (Sigma, St. Louis, $\mathrm{MO}$ ). Protein concentrations were quantified with a detergent-compatible protein assay kit (Bio-Rad Laboratories, Hercules, CA) according to the manufacturer's manual. Fifty-microgram protein extracts were denatured in Laemmli buffer containing $5 \% \beta$-mercaptoethanol, then loaded and separated by gel electrophoresis on a $7 \%$ Bis-Tris gel (Invitrogen, Carlsbad, CA). Primary antibody was incubated at $4^{\circ} \mathrm{C}$ overnight under shaking conditions. Immunoreactive bands were visualized on nitrocellulose membranes using alkaline phosphatase-linked anti-mouse or rabbit antibody and the ECF detection system with a Phosphorlmager (GE Healthcare, Piscataway, NJ). The densities of bands were quantified with the Phosphorlmager. Anti-STAT3, anti-phospho-STAT3 (Tyr705), antiPSTAT1, and anti-STAT1 antibodies were purchased from Cell Signaling Technology (Beverly, MA). Mouse monoclonal anti-actin antibody was obtained from Sigma-Aldrich. 


\section{Real-Time PCR}

Real-time PCR was used to determine the expression of proinflammatory cytokines and inflammatory markers in the liver. Total RNA was purified from 30-mg liver samples according to the manufacturer (Qiagen, Valencia, CA), and then $1 \mu \mathrm{g}$ of mRNA was reverse-transcribed to cDNA using a High Capacity cDNA Reverse Transcription kit (Invitrogen). cDNA template was diluted 1:5 and amplified in realtime PCR using iTaq SYBR Green Supermix (Bio-Rad). An initial denaturation at $95^{\circ} \mathrm{C}$ for 3 minutes was followed with PCR cycling: $95^{\circ} \mathrm{C}\left(15\right.$ seconds) and $58^{\circ} \mathrm{C}$ (30 seconds) for 40 cycles. Relative mRNA levels were calculated by means of $2^{-\Delta \Delta C T}(\Delta \Delta \mathrm{CT}=$ difference of crossing points of test samples and respective control samples as extracted from amplification curves by the LightCycler software; Roche Diagnostics Corporation, Indianapolis, IN) after normalization to $18 \mathrm{~S}$ expression used as an internal standard. Fold inductions of analyzed mRNA expression were normalized on 18S RNA expression. The sequence of primer was described previously. ${ }^{18}$

\section{Flow Cytometry Analysis}

Liver lymphocytes were isolated as described previously ${ }^{20}$ and analyzed by a fluorescence-activated cell cytometer (FACScalibur; Becton Dickinson) using anti-NK1.1, antiCD3, anti-F4/80, anti-Gr1, and anti-CD11b antibodies

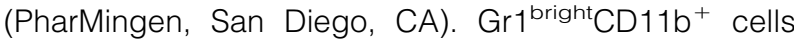
mainly represent neutrophils, whereas $\mathrm{F} 4 / 80^{+}$cells represent macrophages.

\section{Statistical Analysis}

Data are expressed as means \pm SEM. To compare values obtained from two groups, the Student's $t$-test was performed. To compare values obtained from three or more groups, onefactor analysis of variance was used, followed by Tukey's post hoc test. Values represent means \pm SEM ( $n=6$ to 12). Statistical significance was taken at the $P<0.05$ level.

\section{Results}

\section{IL-10 Is Up-Regulated in the Liver and Spleen after PHx via a TLR4-Dependent Manner}

To determine the regulation of $\mathrm{LL}-10 \mathrm{mRNA}$ levels during the early stage of liver regeneration, real-time PCR analyses were performed on the liver and spleen mRNAs after PHx. As shown in Figure 1, $A$ and $B$, expression of IL-10 mRNA in the liver and spleen was significantly increased, with peak effect occurring at 1 hour and 3 hours, but returned to basal levels by 9 hours after $\mathrm{PHx}$, which is consistent with a previous report. ${ }^{21}$ In contrast, no changes in IL-10 mRNA expression were observed in sham-operated mice. Interestingly, expression of IL-6 mRNAs in the liver and spleen were up-regulated in mice from both $\mathrm{PHx}$ and sham-operated groups. TNF- $\alpha$ mRNA levels were slightly increased only at 1 hour in the liver after $\mathrm{PHx}$, and there was no change in the sham-operated control group.
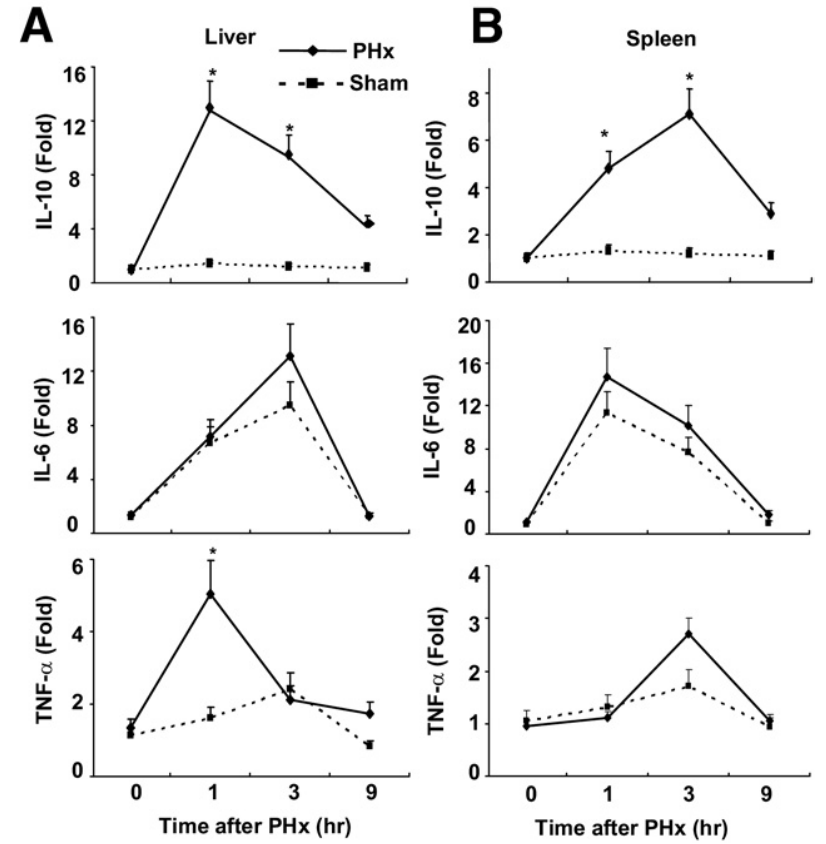

Figure 1. Up-regulation of proinflammatory cytokines in the liver and spleen after PHx. A and B: C57BL/6 mice were subject to PHx. Liver (A) and spleen (B) tissues were then collected for real-time PCR analyses. The value from 0 hours time point was set as onefold. ${ }^{*} P<0.01$ in comparison with corresponding sham controls.

To further investigate whether the increased IL-10 production after $\mathrm{PHx}$ is dependent on the TLR4 signaling pathway, we compared the mRNA expression of IL-10 in TLR4 mutant mice and wild-type mice after $\mathrm{PHx}$ or sham operation. As shown in Figure 2, the levels of IL-10 mRNA in the liver and spleen were significantly up-regulated in wild-type mice after $\mathrm{PHx}$; such induction was markedly diminished in TLR4 mutant mice. Induction of $\mathrm{TNF}-\alpha$, but not IL-6, was diminished in TLR4 mutant mice compared with wild-type mice. Sham operation did not up-regulate IL-10 and TNF- $\alpha$ mRNAs in either wild-type or TLR4 mutant mice, but induced similar elevation of IL-6 mRNA in the liver and spleen from both strains of mice.

\section{Enhanced Inflammatory Response in IL-10 ${ }^{-/-}$ Mice after PHx}

To explore whether IL-10 production plays a role in controlling liver inflammation after $\mathrm{PHx}$, the mRNA expression of several proinflammatory cytokines and inflammatory markers of macrophages (F4/80) and monocytes (CCR2) was examined in wild-type and IL-10-1- mice. As illustrated in Figure 3A, expression of TNF- $\alpha$ and IL-6 was significantly up-regulated in wild-type mice after $\mathrm{PHx}$, and such upregulation was more profound in $\mathrm{IL}-10^{-1-}$ mice. Expression of IFN- $\gamma, \mathrm{F} 4 / 80, \mathrm{CCR} 2$, and MCP-1 was only slightly elevated in wild-type mice after $\mathrm{PHx}$, but was markedly elevated in IL-10 $10^{-1-}$ mice, with 40- to 60-fold induction of F4/80 mRNA, 20- to 70-fold induction of CCR2, and 80-fold induction of MCP-1. Next, we determined the protein levels of several proinflammatory cytokines in the liver tissues from wild-type and $\mathrm{IL}-10^{-1-}$ mice after $\mathrm{PHx}$. As illustrated in 

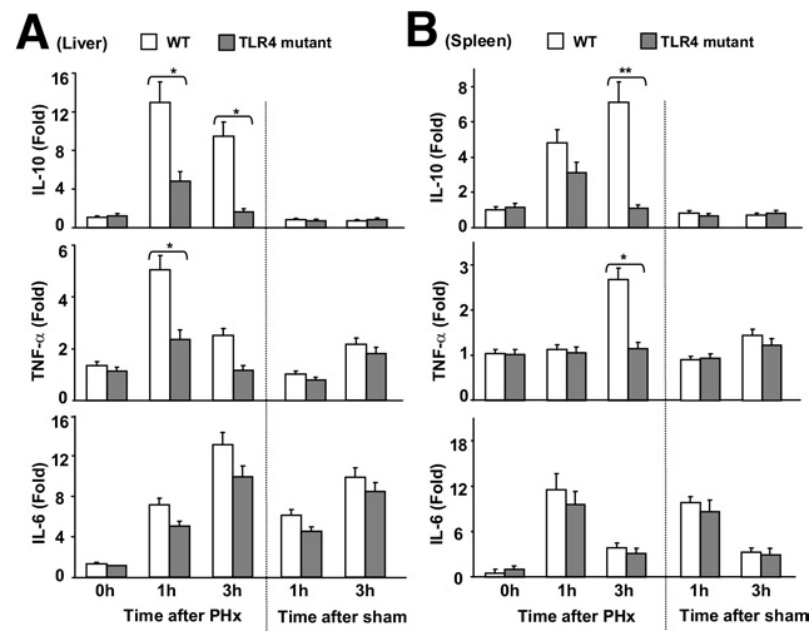

Figure 2. Up-regulation of IL-10 and TNF- $\alpha$ in the liver and spleen after PHx is dependent on TLR4. A and B: TLR4 mutant and wild-type (WT) control mice were subject to PHx or sham operation. Liver (A) and spleen (B) tissues were then collected for real-time PCR analyses. The value from 0 hours time point was set as onefold. ${ }^{*} P<0.05$; ${ }^{* * *} P<0.01$.

Figure 3B, levels of TNF- $\alpha$, IL-6, IFN- $\gamma$, and MCP-1 proteins in the liver were higher in $\mathrm{IL}-10^{-1-}$ mice 3 hours after $\mathrm{PHx}$ when compared with the levels in wild-type mice.

To further confirm the increased inflammatory response in $\mathrm{IL}-10^{-1-}$ mice, liver lymphocytes were isolated and analyzed by flow cytometry. As shown in Figure 3C, the number of neutrophils and macrophages in the liver was higher in IL-

\section{A}

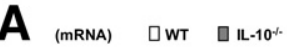
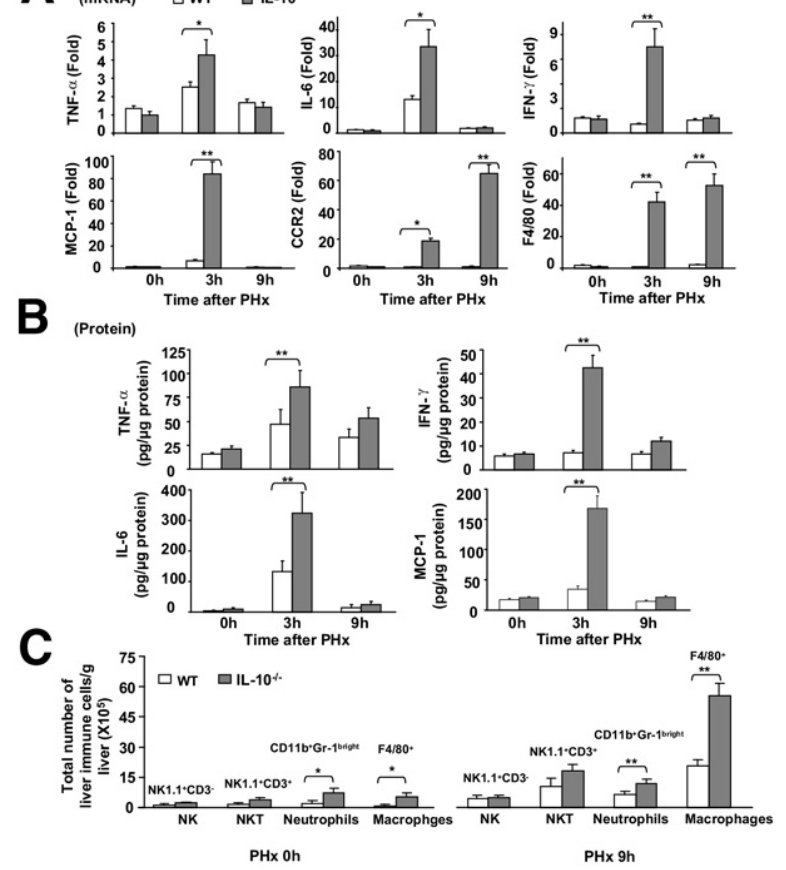

Figure 3. Enhanced liver inflammation after PHx in $\mathrm{IL}-10^{-/-}$mice. $\mathbf{A}$ and $\mathbf{B}$ : IL-10 $0^{-/-}$and wild-type (WT) control mice were subject to PHx. Liver tissues were collected. RNA and protein extractions were then prepared for real-time PCR (A) and cytokines analyses (B), respectively. The value from 0 hours time point in $\mathbf{A}$ was set as onefold. C: Liver lymphocytes were isolated and analyzed by flow cytometry before or after PHx, and the number of liver lymphocytes was quantified. ${ }^{*} P<0.05,{ }^{* * *} P<0.01$.
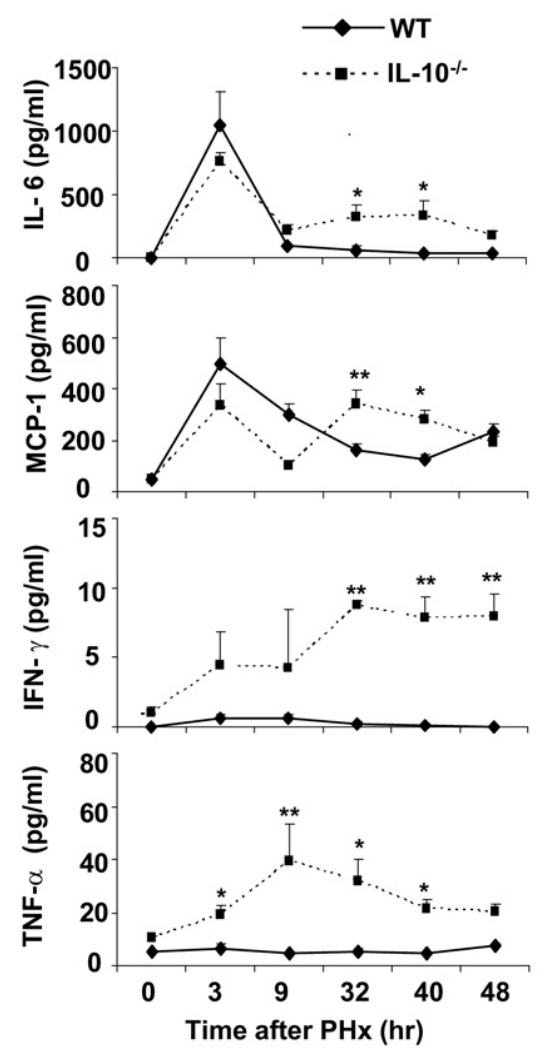

Figure 4. Up-regulation of proinflammatory cytokines in the serum after PHx in IL- $10^{-1-}$ mice. IL- $10^{-1-}$ and wild-type (WT) control mice were subject to PHx. Serum were collected, and the levels of proinflammatory cytokines were measured. ${ }^{*} P<0.05 ;{ }^{* *} P<0.01$ in comparison with corresponding wild-type controls.

$10^{-1-}$ mice before $\mathrm{PHx}$ than in wild-type mice. After $\mathrm{PHx}$, the number of neutrophils and macrophages in the liver was elevated in both wild-type and IL-10 $10^{-1-}$ mice, with a higher number in the later group. The number of NKT cells was similarly elevated in both wild-type and IL-10-/- mice after PHx.

To further confirm the increased inflammatory response in $\mathrm{IL}-10^{-1-}$ mice after $\mathrm{PHx}$, we examined the protein levels of several proinflammatory cytokines in the serum of wildtype and $\mathrm{IL}-10^{-1-}$ mice after PHx. As illustrated in Figure 4, serum levels of IL-6 and MCP-1 were significantly higher in $\mathrm{IL}-10^{-/-}$mice compared with wild-type mice 32 hours and 40 hours after PHx. Serum levels of IFN- $\gamma$ and TNF- $\alpha$ were not elevated in wild-type mice after $\mathrm{PHx}$ but were markedly increased in $\mathrm{IL}-10^{-1-}$ mice.

\section{Deletion of the IL-10 Gene Enhances Liver Regeneration with Elevated Hepatic STAT3 Activation}

To understand the role of anti-inflammatory cytokine IL-10 in regulating liver regeneration, we compared the hepatocyte proliferation after $\mathrm{PHx}$ between wild-type and $\mathrm{IL}-10^{-/-}$ mice. After $\mathrm{PHx}, \mathrm{IL}-10^{-/-}$and wild-type mice showed no obvious adverse phenotype, no mortality, and no obvious hepatocyte apoptosis. Compared with wildtype mice, IL-10 ${ }^{-1-}$ mice had significantly higher numbers of $\mathrm{BrdU}^{+}$hepatocytes 48 hours and 60 hours after 

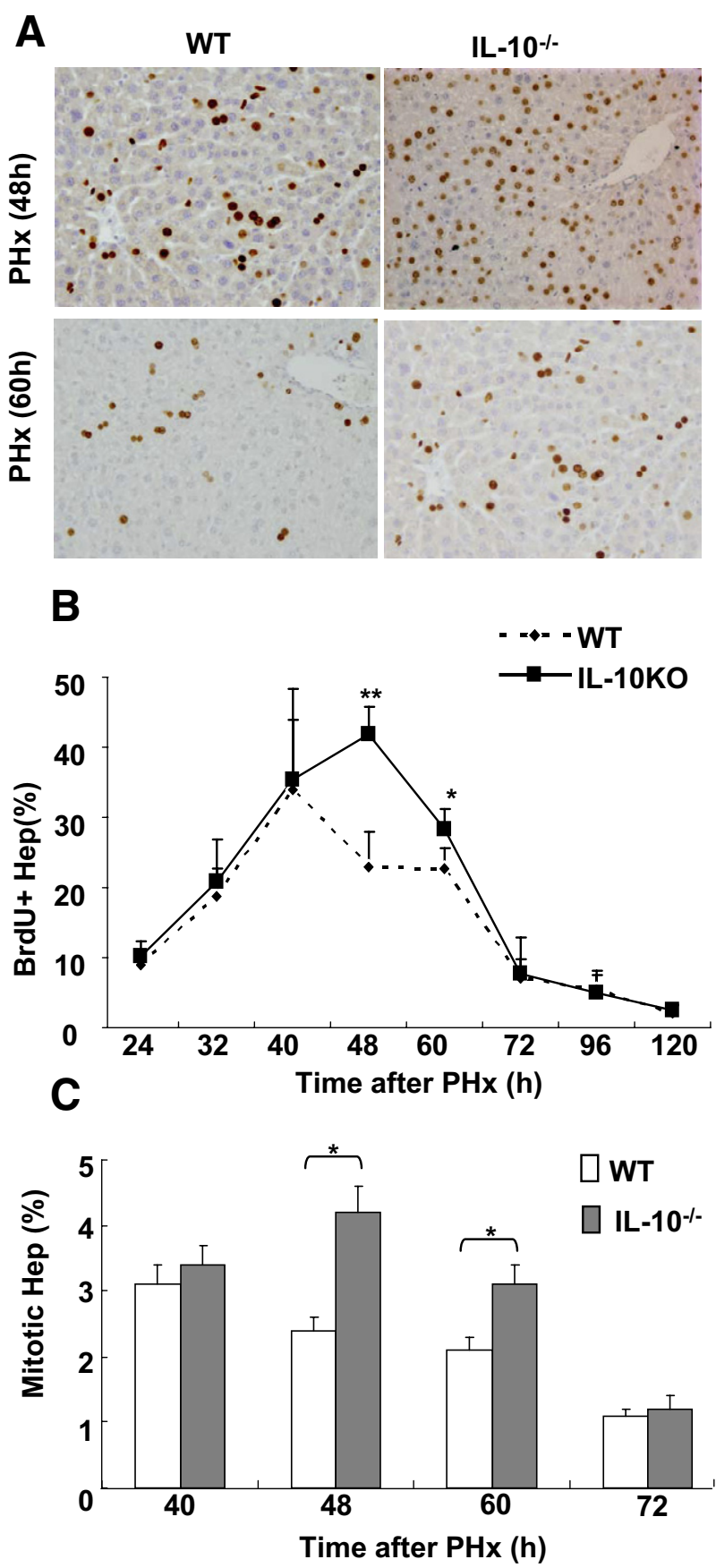

Figure 5. Enhanced liver regeneration after $\mathrm{PHx}$ in $\mathrm{IL}-10^{-/-}$mice. A, B, and C: IL-10 ${ }^{-1-}$ and wild-type (WT) control mice were subject to PHx. Liver regeneration was measured by $\mathrm{BrdU}^{+}$incorporation assay. Representative image of BrdU staining is shown in $\mathbf{A}$, the percentage of $\mathrm{BrdU}^{+}$hepatocytes is shown in $\mathbf{B}$, and the percentage of mitotic hepatocytes is shown in $\mathbf{C} .{ }^{*} P$ $<0.05 ;{ }^{* * *} P<0.01$

$\mathrm{PHx}$, whereas the numbers of $\mathrm{BrdU}^{+}$hepatocytes were similar between these two groups at other time points (Figure 5). The liver/body weight ratios after $\mathrm{PHx}$ were slightly higher but did not reach statistical difference in $\mathrm{IL}-10^{-/-}$mice compared with wild-type mice (data not shown). The number of $\mathrm{BrdU}^{+}$hepatocytes was similar in sham-operated wild-type and $1 \mathrm{~L}-10^{-1-}$ mice (data not shown). Finally, the number of mitotic hepatocytes was higher in $\mathrm{IL}-10^{-1-}$ mice compared with wild-type mice 48 hours and 60 hours after PHx (Figure 5C).

Next, we investigated the mechanisms underlying enhanced liver regeneration in $\mathrm{IL}-10^{-1-}$ mice by analyzing the activation of the STAT3 pathway, which promotes hepatocyte survival and liver regeneration, ${ }^{13-15}$ as well as STAT1 activation, which induces hepatocyte apoptosis and inhibits liver regeneration. ${ }^{22}$ As illustrated in Figure 6, hepatic STAT3 was activated after PHx in wild-type mice, as reflected by elevated levels of phospho-STAT3 (pSTAT3) with peak effect occurring 3 to 6 hours after surgery. The expression levels of pSTAT3 were significantly higher 3 and 6 hours after $\mathrm{PHx}$ and remained higher 32 to 40 hours after $\mathrm{PHx}$ in $\mathrm{IL}-10^{-1-}$ mice than in wild-type mice. In contrast, expression of hepatic PSTAT1 was not detected in wild-type mice and detected at very low levels in IL-10 ${ }^{-1-}$ mice 32 to 60 hours after $\mathrm{PHx}$.

\section{An Additional Deletion of STAT3 in Hepatocytes Decreases Liver Regeneration in IL-10 ${ }^{-1-}$ Mice after $\mathrm{PHX}$}

The above data show that STAT3 activation is enhanced in $\mathrm{IL}-10^{-1-}$ mice after PHx. Since hepatic STAT3 has been shown to play an important role in promoting liver regeneration, ${ }^{13-15}$ we hypothesized that enhanced
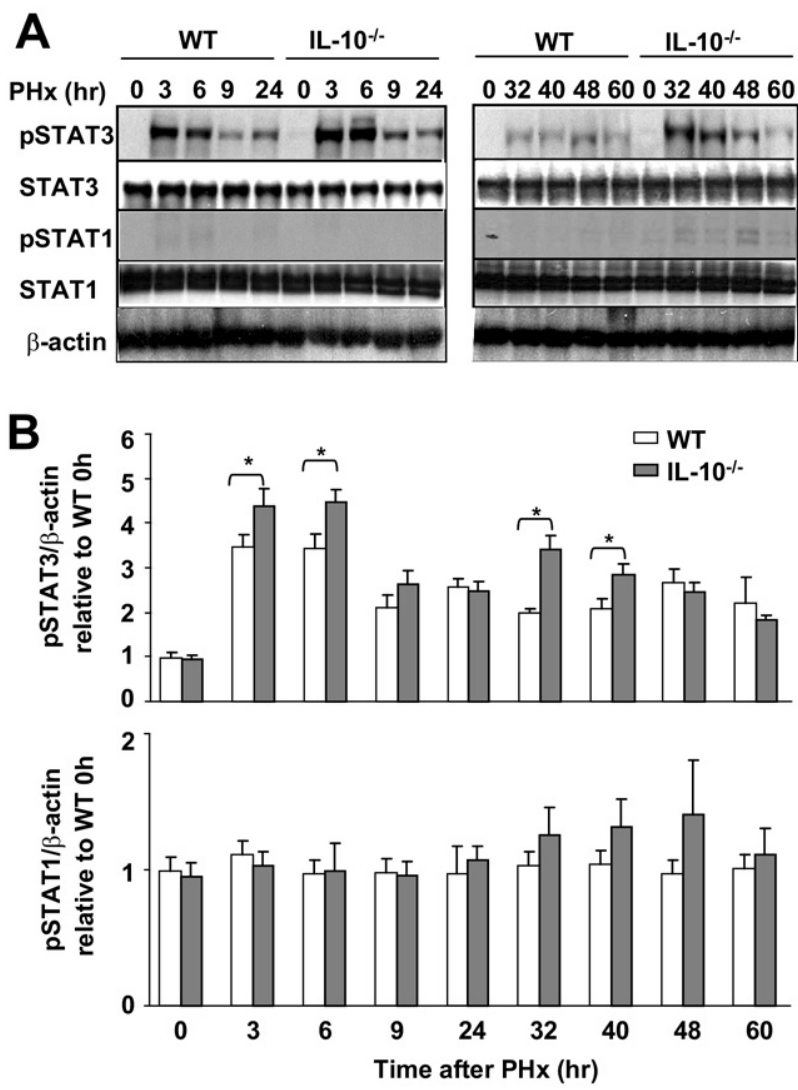

Figure 6. Enhanced hepatic pSTAT3 activation after PHx in $\mathrm{IL}-10^{-/-}$mice $\mathbf{A}$ and B: IL- $10^{-/-}$and wild-type (WT) control mice were subject to PHx. Liver tissues were collected for Western blot analyses. Representative blots from three independent experiments are shown in $\mathbf{A}$, and densitometric analysis of immunoblots is shown in $\mathbf{B} .{ }^{*} P<0.05$ 
STAT3 may contribute to the increased liver regeneration in $\mathrm{IL}-10^{-/-}$mice. To test this hypothesis, we made an additional deletion of hepatocyte STAT3 in IL-10 ${ }^{-1-}$ mice to generate STAT3 ${ }^{\mathrm{Hep}-1-} \mid \mathrm{L}-10^{-1-}$ mice.

As shown in Figure 7A, Western blot analyses confirmed the dramatic reduction of STAT3 and PSTAT3 protein expression in the liver of STAT3 ${ }^{\text {Hep-I- }}$ and STAT3 ${ }^{\text {Hep }}{ }^{-1-} \mid \mathrm{L}-10^{-1-}$ mice. The low levels of STAT3 and pSTAT3 expression that were still detected in these hepatocyte-specific STAT3 $\mathrm{KO}\left(\mathrm{STAT3}^{\mathrm{Hep}-1-}\right)$ mice and double KO mice may be due to the expression of STAT3 in nonparenchymal cells in the liver. Consistent with previous reports, ${ }^{13,15}$ STAT3 ${ }^{\mathrm{Hep}-/-}$ mice had increased levels of PSTAT1 and STAT1 expression after PHx com-

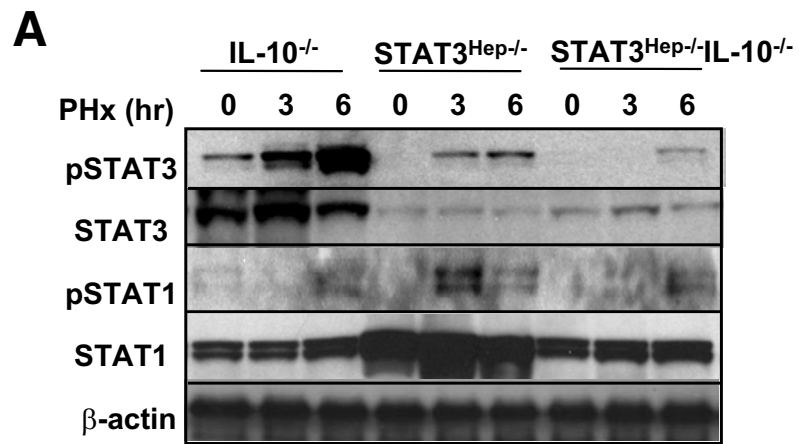

\section{B}
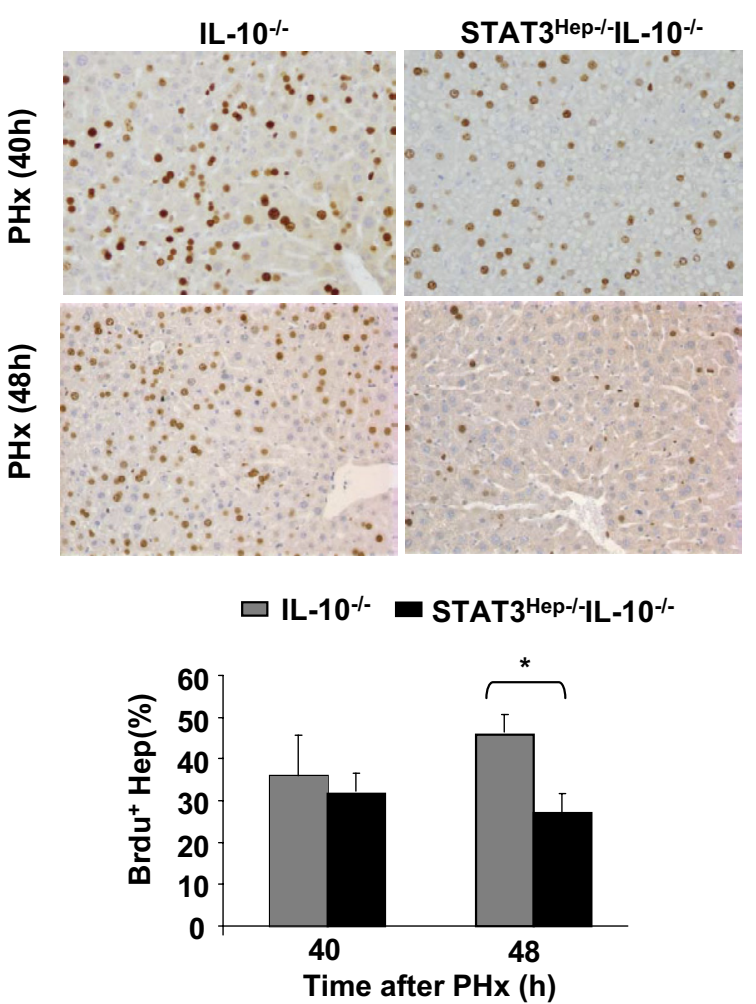

Figure 7. Deletion of hepatocyte STAT3 reduced liver regeneration after PHx in IL-10 $10^{-/-}$mice. A: Western blot analyses of liver extracts were performed from partially hepatectomized mice. Representative blots from three independent experiments are shown. B: Liver regeneration was determined by BrdU incorporation assay. Representative image of BrdU staining is shown in the upper panel, and the percentage of $\mathrm{BrdU}^{+}$hepatocytes is shown in the lower panel. ${ }^{*} P<0.05$. pared with wild-type mice. Interestingly, PSTAT1 expression was not detected in $\mathrm{IL}-10^{-1-}$ mice and only weakly activated after $\mathrm{PH} \mathrm{x}$ in STAT3 ${ }^{\mathrm{Hep}-I-} \mathrm{IL}-10^{-1-}$ mice. After PHx, all STAT3 ${ }^{\mathrm{Hep}-I-} \mid \mathrm{L}-10^{-/-}$mice survived. The number of $\mathrm{BrdU}^{+}$hepatocytes was significantly lower in STAT3 ${ }^{\text {Hep }-1-} \mid \mathrm{L}-10^{-1-}$ mice compared with IL-10 ${ }^{-1-}$ mice 48 hours after $\mathrm{PHx}$ (Figure 7B).

\section{Discussion}

Previous studies have well documented that proinflammatory cytokines TNF- $\alpha$ and IL-6 play an important role in promoting liver regeneration. ${ }^{1-6}$ In the current study, we provide several lines of evidence suggesting that the anti-inflammatory cytokine IL-10 negatively regulates liver regeneration via suppressing proinflammatory response and STAT3 activation in hepatocytes. First, after PHx, expression of IL-10 in the liver and spleen was significantly up-regulated. Second, elevation of proinflammatory cytokines and inflammatory markers after $\mathrm{PHx}$ was higher in $\mathrm{IL}-10^{-/-}$mice than in wild-type mice. Third, $\mathrm{IL}-10^{-/-}$mice had greater liver regeneration than wild-type mice after $\mathrm{PHx}$, which is associated with enhanced STAT3 activation in the liver. Deletion of STAT3 in hepatocytes reduced liver regeneration in $\mathrm{IL}-10^{-1-}$ mice after $\mathrm{PHx}$.

\section{Up-Regulation of IL-10 after PHx in a TLR4-Dependent Manner}

It is generally believed that after PHx, levels of LPS in the liver are elevated, which then stimulate Kupffer cells to produce proinflammatory cytokines such as TNF- $\alpha$ and IL-6 that subsequently prime and promote liver regeneration. ${ }^{1-6}$ However, the role of LPS in induction of IL-6 after $\mathrm{PH} x$ has recently been questioned. ${ }^{23}$ Deletion of the LPS receptor TLR4 gene or its coreceptor CD14 did not affect IL-6 induction at 4 hours after $\mathrm{PHx},{ }^{23}$ which was also confirmed in the present study showing that $\mathrm{PHx}$-mediated induction of IL-6 was comparable in wild-type mice and TLR4 mutant mice (Figure 2). This may not be surprising because virtually all multinucleated cells can produce IL-6 after various stimuli, including stress. Indeed, sham operation (surgical stress) results in elevation of IL-6 (Figure 1) and subsequent activation of STAT3 in the liver, ${ }^{24}$ which are comparable to what is observed after $\mathrm{PHx}$. In contrast, expression of $\mathrm{IL}-10$ in the liver and spleen was only induced after $\mathrm{PHx}$, but not sham operation (Figure 1), and such induction was significantly blunted in TLR4 mutant mice (Figure 2). In contrast to IL-6 that is ubiquitously expressed, IL-10 is mainly produced by macrophages and activated T cells. ${ }^{17}$ Indeed, depletion of Kupffer cells (liver macrophages) has been shown to abolish induction of IL-10 expression in the regenerating liver after $\mathrm{PHx} .{ }^{21}$ Taken together, these findings suggest that after PHx, elevated levels of LPS in the liver stimulate Kupffer cells via targeting TLR4 to produce IL-10 that subsequently inhibits inflammatory response and liver regeneration. 
1620 Yin et al

AJP April 2011, Vol. 178, No. 4

\section{IL-10 Plays an Important Role in Tempering Inflammatory Response after $\mathrm{PHx}$}

IL-10 acts as a potent anti-inflammatory cytokine via selectively blocking the expression of proinflammatory genes in myeloid cells including macrophages and neutrophils activated by pathogen recognition receptor ligands such as LPS. ${ }^{17} \mathrm{IL}-10$ exerts its function via binding IL-10R1 and IL-10R2, followed by activation of STAT3 in myeloid cells. Conditional deletion of STAT3 in myeloid cells results in enhanced inflammatory response and inflammation in various organs including the liver. ${ }^{18,25,26}$ Here, we demonstrate that deletion of IL-10 markedly increased the inflammatory responses in the liver after $\mathrm{PHx}$ (Figure 3). Expression of F4/80 (a marker for macrophages) and CCR2 (a marker for monocytes) in the liver was slightly induced after $\mathrm{PHx}$ in wild-type mice but was induced up to 60-fold in IL-10 ${ }^{-1-}$ mice. In addition, serum and hepatic levels of proinflammatory cytokines, including IL-6, MCP-1, IFN- $\gamma$, and TNF- $\gamma$, were significantly higher in $\mathrm{IL}-10^{-1-}$ mice than in wild-type mice after $\mathrm{PHx}$. Finally, the number of neutrophils and macrophages accumulated in the liver after $\mathrm{PHx}$ was higher in $\mathrm{IL}-10^{-/-}$ mice than in wild-type mice. These findings clearly indicate that IL-10 plays an important role in negatively regulating inflammatory response during liver regeneration.

\section{IL-10 Negatively Regulates Hepatocyte Proliferation and Liver Regeneration via Down-Regulation of STAT3 Activation in Hepatocytes after $\mathrm{PHx}$}

Figure 5 shows that the peak of BrdU incorporation occurs at 48 hours after $\mathrm{PHx}$ in $\mathrm{IL}-10^{-1-}$ mice, which is significantly higher than that which occurs at 40 hours after $\mathrm{PHx}$ in wild-type mice; however, BrdU incorporation was similar at most other time points we measured between these two groups. This suggests that liver regeneration is only modestly increased in $\mathrm{IL}-10^{-1-}$ mice after $\mathrm{PHx}$ compared with wild-type mice. However, IL-10 may play a more important role in controlling hepatocyte proliferation and liver regeneration induced by chronic liver injury and inflammation, in contrast to the PHx model we used here that has rapid liver regeneration with minimal inflammation. Interestingly, although the peak of BrdU incorporation after $\mathrm{PHx}$ is significantly higher in $\mathrm{IL}-10^{-/-}$ mice than in wild-type mice, there is no difference in liver/body weight ratios between these 2 groups. This may be because the liver/body weight ratio is tightly controlled by a wide variety of factors and their downstream signaling pathways. Indeed, it has been reported that hepatocyte proliferation is enhanced or suppressed, whereas liver mass restores normally after $\mathrm{PHx}$ under many conditions, including in rats treated with dexamethasone, ${ }^{27}$ in Skp2-deficient mice, ${ }^{28}$ and in hepatic $\beta$-catenin conditional knockout mice. ${ }^{29}$ At present, the mechanisms by which IL-10 $10^{-1-}$ mice had enhanced hepatocyte proliferation but similar liver mass restoration remain unknown. Terminal deoxynucleotidyl transferasemediated dUTP nick-end labeling (TUNEL) analyses show a similar number of apoptotic hepatocytes in wildtype mice and $\mathrm{IL}-10^{-1-}$ mice (data not shown), suggesting that the similar liver mass restoration despite enhanced hepatocyte proliferation in $\mathrm{IL}-10^{-1-}$ mice is not due to enhanced hepatocyte apoptosis. Previous studies reported that STAT3 plays an important role in constraining hepatocyte size after $\mathrm{PHx} .{ }^{14,30}$ Since $\mathrm{IL}-10^{-/-}$mice had higher levels of hepatic STAT3 activation than wildtype mice, it is plausible to speculate that $\mathrm{IL}-10^{-1-}$ mice have a smaller size of hepatocytes than wild-type mice after $\mathrm{PHx}$. Future studies are required to confirm this speculation. Additionally, recent studies show that integrin-linked kinase, which is involved in transmission of the extracellular matrix (ECM) signaling by way of integrin receptors, plays an important role in termination of regeneration after $\mathrm{PHx} .^{7,31}$ It would be interesting to examine whether IL-10 affects integrin-linked kinase activation and expression in the liver, thereby affecting the termination of liver regeneration.

The enhancement of liver regeneration caused by IL-10 deficiency may be due to elevation of the inflammatory response and subsequent activation of STAT3 in the liver, because many of these inflammatory cytokines (such as TNF- $\alpha, \mathrm{IL}-6$, and MCP-1) and their downstream signaling molecules have been shown to promote liver regeneration. ${ }^{1-5,10,11,32}$ Furthermore, an additional deletion of hepatocyte STAT3 in IL-10 ${ }^{-1-}$ mice markedly reduced liver regeneration in these mice (Figure 7 ), suggesting that elevation of inflammatory response-associated STAT3 activation in the liver contributes to the enhanced liver regeneration in $\mathrm{IL}-10^{-1-}$ mice.

It is known that the anti-inflammatory action of $\mathrm{IL}-10$ is mainly mediated via activation of STAT3 signaling pathway in myeloid cells including macrophages and neutrophils. ${ }^{33}$ Deletion of the STAT3 gene in myeloid cells (STAT3 ${ }^{\mathrm{Mye-}-1-}$ mice) results in many similar phenotypes as in $\mathrm{IL}-10^{-1-}$ mice, including enhanced inflammatory response and liver regeneration after $\mathrm{PHx},{ }^{13}$ suggesting that the negative effect of IL-10 on inflammation and liver regeneration after $\mathrm{PHx}$ is mediated, at least in part, via $\mathrm{IL}-10$ activation of STAT3 in myeloid cells. Despite elevation of liver inflammation and proapoptotic cytokine IFN- $\gamma$ in $\mathrm{IL}-10^{-1-}$ mice (in this study) and STAT3 ${ }^{\mathrm{Mye}-1-}$ mice, ${ }^{13}$ no mortality and no obvious hepatocyte apoptosis were observed in these mice after $\mathrm{PHx}$. This is probably due to the enhanced activation of cell survival signal molecule STAT3 in the livers of IL-10 mice (Figure 6) and STAT3 ${ }^{\mathrm{Mye}-1-}$ mice. ${ }^{13}$ Interestingly, an additional deletion of hepatocyte STAT3 in STAT3 ${ }^{\text {Mye-l- }}$ mice (STAT3 ${ }^{\mathrm{Hep}-1-}$ STAT3 ${ }^{\mathrm{Mye}-1-}$ double $\mathrm{KO}$ mice) results in massive apoptosis and high mortality after $\mathrm{PHx}$, which is likely due to a dramatic increase in proapoptotic signal STAT1 activation in hepatocytes in these double KO mice. ${ }^{13}$ Surprisingly, an additional deletion of hepatic STAT3 in IL$10^{-1-}$ mice did not cause hepatocyte apoptosis and mortality after $\mathrm{PHx}$, which may be because the expression of proapoptotic STAT1 signal in the liver was only slightly elevated in these STAT3 ${ }^{\mathrm{Hep}-1-} \mathrm{IL}-10^{-1-}$ double KO mice (Figure 7). At present, it is not clear why hepatic STAT1 signal was markedly activated in STAT3 ${ }^{\mathrm{Hep}-1-}$ STAT3 $^{\mathrm{Mye}-1-}$ double $\mathrm{KO}$ mice but only slightly elevated in STAT3 ${ }^{\mathrm{Hep}-1-} \mathrm{IL}-$ $10^{-1-}$ double $\mathrm{KO}$ mice despite similar elevation of serum 
levels of IFN- $\gamma$, a major cytokine to activate STAT1, in both strains of double KO mice after PHx (Figure 4).$^{13}$ Further studies are required to clarify this mechanism.

In summary, in addition to its important role in the antiinflammatory effects in the regenerating liver, IL-10 coordinates with other proinflammatory cytokines to precisely regulate and synchronize hepatocyte proliferation during liver regeneration. IL-10 acts as a repressor in liver regeneration via limiting inflammatory cytokine response and subsequently tempering hepatic STAT3 activation. Elevated levels of serum and hepatic IL-10 have been found in patients with various liver diseases, ${ }^{34,35}$ which may play an important role not only in reducing liver inflammation but also in tempering liver over-regeneration stimulated by inflammatory response without causing liver tumor transformation.

\section{References}

1. Michalopoulos GK: Liver regeneration after partial hepatectomy: critical analysis of mechanistic dilemmas. Am J Pathol 2010, 176:2-13

2. Michalopoulos GK: Liver regeneration. J Cell Physiol 2007, 213:286300

3. Fausto N, Campbell JS, Riehle KJ: Liver regeneration. Hepatology 2006, 43:S45-S53

4. Taub R: Liver regeneration: from myth to mechanism. Nat Rev Mol Cell Biol 2004, 5:836-847

5. Fausto N: Liver regeneration and repair: hepatocytes, progenitor cells, and stem cells. Hepatology 2004, 39:1477-1487

6. Koniaris LG, McKillop IH, Schwartz SI, Zimmers TA: Liver regeneration. J Am Coll Surg 2003, 197:634-659

7. Apte U, Gkretsi V, Bowen WC, Mars WM, Luo JH, Donthamsetty S, Orr A, Monga SP, Wu C, Michalopoulos GK: Enhanced liver regeneration following changes induced by hepatocyte-specific genetic ablation of integrin-linked kinase. Hepatology 2009, 50:844-851

8. Cornell RP: Gut-derived endotoxin elicits hepatotrophic factor secretion for liver regeneration. Am J Physiol 1985, 249:R551-R562

9. Xu CP, Liu J, Liu JC, Han DW, Zhang Y, Zhao YC: Dynamic changes and mechanism of intestinal endotoxemia in partially hepatectomized rats. World J Gastroenterol 2007, 13:3592-3597

10. Cressman DE, Greenbaum LE, DeAngelis RA, Ciliberto G, Furth EE, Poli V, Taub R: Liver failure and defective hepatocyte regeneration in interleukin-6-deficient mice. Science 1996, 274:1379-1383

11. Yamada Y, Kirillova I, Peschon JJ, Fausto N: Initiation of liver growth by tumor necrosis factor: deficient liver regeneration in mice lacking type I tumor necrosis factor receptor, Proc Natl Acad Sci U S A 1997, 94:1441-1446

12. Gao B: Cytokines, STATs and liver disease. Cell Mol Immunol 2005 2:92-100

13. Wang H, Park O, Lafdil F, Shen K, Horiguchi N, Yin S, Fu XY, Kunos G, Gao B: Interplay of hepatic and myeloid signal transducer and activator of transcription 3 in facilitating liver regeneration via tempering innate immunity. Hepatology 2010, 51:1354-1362

14. Haga S, Ozaki M, Inoue H, Okamoto Y, Ogawa W, Takeda K, Akira S, Todo S: The survival pathways phosphatidylinositol-3 kinase (PI3-K)/ phosphoinositide-dependent protein kinase 1 (PDK1)/Akt modulate liver regeneration through hepatocyte size rather than proliferation. Hepatology 2009, 49:204-214

15. Li W, Liang X, Kellendonk C, Poli V, Taub R: STAT3 contributes to the mitogenic response of hepatocytes during liver regeneration. $J$ Bio Chem 2002, 277:28411-28417

16. Campbell JS, Prichard L, Schaper F, Schmitz J, Stephenson-Famy A Rosenfeld ME, Argast GM, Heinrich PC, Fausto N: Expression of suppressors of cytokine signaling during liver regeneration. J Clin Invest 2001, 107:1285-1292
17. Mosser DM, Zhang X: Interleukin-10: new perspectives on an old cytokine. Immunol Rev 2008, 226:205-218

18. Horiguchi N, Wang L, Mukhopadhyay P, Park O, Jeong WI, Lafdil F, Osei-Hyiaman D, Moh A, Fu XY, Pacher P, Kunos G, Gao B: Cell type-dependent pro- and anti-inflammatory role of signal transducer and activator of transcription 3 in alcoholic liver injury. Gastroenterology 2008, 134:1148-1158

19. Mitchell $\mathrm{C}$, Willenbring $\mathrm{H}$ : A reproducible and well-tolerated method for 2/3 partial hepatectomy in mice. Nat Protoc 2008, 3:1167-1170

20. Liu ZX, Han D, Gunawan B, Kaplowitz N: Neutrophil depletion protects against murine acetaminophen hepatotoxicity. Hepatology 2006, 43:1220-1230

21. Rai RM, Loffreda S, Karp CL, Yang SQ, Lin HZ, Diehl AM: Kupffer cell depletion abolishes induction of interleukin-10 and permits sustained overexpression of tumor necrosis factor alpha messenger RNA in the regenerating rat liver. Hepatology 1997, 25:889-895

22. Sun R, Park O, Horiguchi N, Kulkarni S, Jeong WI, Sun HY, Radaeva S, Gao B: STAT1 contributes to dsRNA inhibition of liver regeneration after partial hepatectomy in mice. Hepatology 2006, 44:955-966

23. Campbell JS, Riehle KJ, Brooling JT, Bauer RL, Mitchell C, Fausto N: Proinflammatory cytokine production in liver regeneration is Myd88dependent, but independent of Cd14, TIr2, and TIr4, J Immunol 2006, 176:2522-2528

24. Heim MH, Gamboni G, Beglinger C, Gyr K: Specific activation of AP-1 but not Stat3 in regenerating liver in mice. Eur J Clin Invest 1997, 27:948-955

25. Lafdil F, Wang H, Park O, Zhang W, Moritoki Y, Yin S, Fu XY, Gershwin ME, Lian ZX, Gao B: Myeloid STAT3 inhibits T cell-mediated hepatitis by regulating $T$ helper 1 cytokine and interleukin-17 production, Gastroenterology 2009, 137:2125-2135.e1-e2

26. Horiguchi N, Lafdil F, Miller AM, Park O, Wang H, Rajesh M, Mukhopadhyay $\mathrm{P}, \mathrm{Fu} X Y$, Pacher $\mathrm{P}$, Gao B: Dissociation between liver inflammation and hepatocellular damage induced by carbon tetrachloride in myeloid cell-specific signal transducer and activator of transcription 3 gene knockout mice. Hepatology 2010, 51:1724-1734

27. Nagy P, Teramoto T, Factor VM, Sanchez A, Schnur J, Paku S, Thorgeirsson SS: Reconstitution of liver mass via cellular hypertrophy in the rat. Hepatology 2001, 33:339-345

28. Minamishima YA, Nakayama K, Nakayama K: Recovery of liver mass without proliferation of hepatocytes after partial hepatectomy in Skp2deficient mice. Cancer Res 2002, 62:995-999

29. Zhang XF, Tan X, Zeng G, Misse A, Singh S, Kim Y, Klaunig JE, Monga SP: Conditional beta-catenin loss in mice promotes chemical hepatocarcinogenesis: role of oxidative stress and platelet-derived growth factor receptor alpha/phosphoinositide 3-kinase signaling. Hepatology 2010, 52:954-965

30. Haga S, Ogawa W, Inoue H, Terui K, Ogino T, Igarashi R, Takeda K, Akira S, Enosawa S, Furukawa H, Todo S, Ozaki M: Compensatory recovery of liver mass by Akt-mediated hepatocellular hypertrophy in liver-specific STAT3-deficient mice. J Hepatol 2005, 43:799-807

31. Gkretsi V, Apte U, Mars WM, Bowen WC, Luo JH, Yang Y, Yu YP, Orr A, St-Arnaud R, Dedhar S, Kaestner KH, Wu C, Michalopoulos GK: Liver-specific ablation of integrin-linked kinase in mice results in abnormal histology, enhanced cell proliferation, and hepatomegaly. Hepatology 2008, 48:1932-1941

32. Hennings DL, Mitchell K: Monocyte chemoattractant protein (MCP-1) is required for liver regeneration (abstract). J Invest Med 2010, 58:219

33. Bazzoni FTN, Rossato M, Cassatella MA: Understanding the molecular mechanisms of the multifaceted IL-10-mediated anti-inflammatory response: lessons from neutrophils. Eur J Immunol 2010, 40: 2360-2368

34. Wu JF, Wu TC, Chen $\mathrm{CH}$, Ni YH, Chen HL, Hsu HY, Chang MH: Serum levels of interleukin-10 and interleukin-12 predict early, spontaneous hepatitis B virus e antigen seroconversion, Gastroenterology 2010, 138:165-172 e161-163

35. Abel M, Sene D, Pol S, Bourliere M, Poynard T, Charlotte F, Cacoub $P$, Caillat-Zucman S: Intrahepatic virus-specific IL-10-producing CD8 $T$ cells prevent liver damage during chronic hepatitis $C$ virus infection. Hepatology 2006, 44:1607-1616 